testicular atrophy, and the affected females had a high incidence of obstetric abnormalities. This family also shows that obstetric abnormalities (other than hydramnios) are the result of the maternal and not the fetal genotype, and that these abnormalities are not responsible for the features of congenital myotonic dystrophy.

Dr Harper was well chosen to write this monograph which can be highly recommended for its very thorough cover of a most curious disease. An extra bonus is that the style is lucid and easy to read. My own feeling is that when the conundrum of congenital myotonic dystrophy is solved the following editions of the book will be even more fascinating than the first. In the meantime, this first edition should be available to all paediatricians and neurologists and hopefully will stimulate further research.

SARAH Bundey

\section{Advances in Human Genetics}

Volume 9. Edited by $\mathrm{H}$ Harris and $\mathrm{K}$ Hirschhorn. (Pp xiv +379 ; figures + tables. $\$ 35 \cdot 00$.)

New York, London: Plenum Press. 1979.

Advances in Human Genetics, now in volume 9, is the most important annual publication in human genetics. This year Opitz, Herrmann, Adinolfi, Harnden, et al have contributed reviews of $\alpha$ fetoprotein, chromosomes and neoplasia, malformations in man, aspects of the molecular biology of haemoglobin, and the metabolism of folate.

In an informative chapter on malformation in man Opitz and his colleagues discuss terminology. They denigrate, with justification, the clinician's ignorance and lack of epistomological skill in assigning birth defects to their natural biological subgroups. They reject with emphasis "the deplorable and redundant term anomalad ... on linguistic, biological and historical grounds", but whether their preferred "developmental field complex" will fare any better is uncertain. Their concern for correct usage of terms is without doubt justified, but the chapter will inhibit most people from describing any new syndrome and perhaps that is as it should be; there are too many old syndromes masquerading under a new name.

In the review of $\alpha$-fetoprotein, Adinolfi comprehensively covers the physicochemical properties of the substance, as well as its clinical usefulness in the detection of neoplasia and neural tube defects. He lists other conditions where raised levels have been found and although uncomplicated hydrocephalus heads the list, the significance of raised levels in this latter condition must be doubted. It is traditional to review one metabolic condition, and this year it is folic acid. For the clinician, the main interest is the emphasis the author places on defects of folate metabolism as a cause of neurodegenerative disease in the absence of severe anaemia.

The relationship between chromosomal anomalies and neoplasia is intriguing. There are those genetic diseases which predispose to the development of neoplasia and these are discussed by Harnden, but it is the genetic change within the neoplastic cell that is his main concern. Finally, the chapter on the expression of human haemoglobin loci is an update which substantiates the many claims that haemoglobin is in advance of any other substance in its contribution to the understanding of molecular genetics. Volume 9 is highly recommended.

M BARAITSER

Inherited Disorders of Carbohydrate Metabolism

Edited by D Burman, J B Holton, and

C A Pennock. (Pp xiv +433 ;

figures + tables. $£ 15 \cdot 95$.)

Lancaster: MTP Press. 1979.

This monograph is a report of the proceedings of the annual meeting of the Society for the Study of Inborn Errors of Metabolism (SSIEM) in Bristol in July 1978, and the contributors form a most distinguished international cast. The membership of the society is open to workers in many disciplines, including biochemists and clinicians, and the papers reflect this heterogeneity.

Professor Hers in the Milner lecture gives a masterly account of the control of carbohydrate metabolism in the liver to which he has contributed so much.

Galactosaemia is reviewed in detail. The biochemistry, clinical aspects, problems of pregnancy, prenatal diagnosis, and screening are all covered. However the discussion failed to resolve why patients in Los Angeles have a mean IQ of 95 whereas those treated in the UK have a mean IQ that is approximately 30 points lower even when it is known that dietary control has been 'satisfactory'.

Fructosaemia has a frequency of about 1 in 20000 in Switzerland but I doubt that the diagnosis is made with frequency in the UK. Similarly, patients with fructose 1-6 diphosphatase deficiency probably remain undiagnosed and the chapters on fructose metabolism are a useful summary of both clinical and biochemical aspects of the disorders.

Congenital lactic acidosis remains one of the most 
difficult areas for those concerned with inherited metabolic disease. The literature is confusing and the section on pyruvate metabolism is excellent because contributors have tried to piece together what is known. Biochemical and clinical problems are discussed but inevitably many questions remain unanswered. For instance, why do not all patients with pyruvate carboxylase deficiency have hypoglycaemia after a long fast like those with fructose 1-6 diphosphatase deficiency?

The diagnosis and management of glycogen storage disease is discussed in detail by several contributors including a comprehensive review by Professor Fernandes in the F $\mathbf{P}$ Hudson Memorial Lecture. Finally, some genetic aspects of diabetes are discussed.

The papers in this book cover both the biochemical and clinical aspects of inborn errors of carbohydrate metabolism, both of which are essential for the optimal care of our patients. I can strongly recommend this book to all those who work in this field.

J V LEONARD

Human Genetics. Problems and Approaches

By F Vogel and A G Motulsky.

(Pp xxviii +700 ; figures + tables. $\$ 53 \cdot 90$, DM98.)

Berlin, Heidelberg, New York: Springer Verlag. 1979.

This outstanding book is a landmark in the development of human genetics. For the first time it has proved possible to combine a rigorous and scientific treatment of the subject with a medical approach that makes full use of the wealth of material available from human inherited disease. That the result has been successful reflects not only the background of the authors, whose work and interests have spanned a remarkable range of fundamental and clinical genetic problems, but also their ability to produce a book which is readable and unified while at the same time remaining critical and able to deal with subjects in a depth most unusual in a textbook.

The overall pattern of the book is a familiar one, with a historical and philosophical introduction followed by sections on human cytogenetics, formal genetics, gene action, mutation, and population and evolutionary genetics. A separate chapter on Behaviour Genetics reflects a special interest of both authors and the final chapter discusses the present and future applications of human genetics. Within this simple framework is contained an abundance of information which is up-to-date, authoritative, critically assessed, and clearly presented, to such an extent that each chapter ranks as a first-rate review of its subject, while the combined effect of the whole $\stackrel{\vec{D}}{?}$

is without parallel. develops the two 'paradigms' of Mendel and Galton 등 which form the recurring theme of the entire book. $\frac{\bar{c}}{\bar{s}}$ We are shown clearly how the thoughts of early $\vec{\sigma}$ workers led towards these fundamental concepts $\propto$ and how the various branches of human genetics $\stackrel{\infty}{\circ}$ have subsequently been able to develop, not omitting $\overrightarrow{0}$ the disastrous abuses of the subject in Nazi Germany and the Soviet Union.

The chapter on cytogenetics is clear, well balanced, $\stackrel{\text { S }}{\rightarrow}$ and admirably illustrated with recent karyotypes as well as with diagrams. The summaries of clinical ${ }_{\infty}$ phenotypes in the major chromosome disorders are $\stackrel{\infty}{-}$ particularly helpful and there is an excellent section $\vec{y}$ on $\mathrm{X}$ chromosome disorders and $\mathrm{X}$ inactivation. Formal genetics is treated extensively, with a full discussion of linkage, segregation analysis, multi- $\Rightarrow$ factorial inheritance, and polymorphisms, as well as $\overrightarrow{T r}$ a valuable account of twin studies. It is particularly $\mathbb{D}$ in this chapter that the use of detailed clinical examples in conjunction with the fundamental concepts and modes of analysis from basic genetics is $\vec{\bullet}$ seen to have its value. From the viewpoint of the $\oplus_{\infty}$ clinical geneticist the entire subject has a relevance. ${ }^{-}$ which often seems lacking in other books on human genetics.

Among the remaining chapters, two, on mutation and human behavioural genetics, stand out because they deal in depth with subjects which usually re- $\stackrel{\mathbb{Q}}{\complement}$ ceive scant attention. Being the principal areas of $\overrightarrow{\overrightarrow{\vec{O}}}$ interest of one of the authors in particular they are 3 dealt with authoritatively, and the same thoughtful and critical approach is brought to bear on the moreo difficult to analyse social and behavioural problems as on the molecular problems of mutation.

After reading this book one is left with a strong impression that a turning point has been reached 3 . in human and medical genetics. The subject is now clearly a fully grown scientific discipline, with its own fundamental principles, with a wealth of experi-o mental techniques and unique material, and with clear paths of future development. Yet this situation contrasts with the serious ignorance of many, perhaps the majority, of those working within this field, $\sigma$ particularly in the more practical areas of medicaln genetics, where the very term 'genetics' appears often N to be little more than a label of convenience. With ${ }_{\sigma}^{\omega}$ funding increasingly being directed to the applications of the subject rather than its fundamentals, there is a real danger that many workers may bees? seriously ignorant of the foundations of their speciality. For this reason, if for no other, this book should be compulsory reading for everyone working in human genetics, not excepting the more senior 